MATHEMATICS OF COMPUTATION

S 0025-5718(99)01064-9

Article electronically published on February 15, 1999

\title{
ON THE COUPLING OF BEM AND FEM FOR EXTERIOR PROBLEMS FOR THE HELMHOLTZ EQUATION
}

\author{
RUIXIA LI
}

\begin{abstract}
This paper deals with the coupled procedure of the boundary element method (BEM) and the finite element method (FEM) for the exterior boundary value problems for the Helmholtz equation. A circle is selected as the common boundary on which the integral equation is set up with Fourier expansion. As a result, the exterior problems are transformed into nonlocal boundary value problems in a bounded domain which is treated with FEM, and the normal derivative of the unknown function at the common boundary does not appear. The solvability of the variational equation and the error estimate are also discussed.
\end{abstract}

\section{INTRODUCTION}

The purpose of this paper is to couple BEM and FEM for the numerical solution of the exterior boundary value problems

$$
\begin{array}{ll}
\triangle u+k^{2} u=0 & \text { in } \quad \Omega, \\
u=u_{0}(x, y) & \text { on } \quad \Gamma
\end{array}
$$

and

$$
\begin{aligned}
& \triangle u+k^{2} u=0 \quad \text { in } \quad \Omega, \\
& \frac{\partial u}{\partial n}=u_{n}(x, y) \quad \text { on } \quad \Gamma
\end{aligned}
$$

with the Sommerfeld radiation condition

$$
\frac{\partial u}{\partial r}-i k u=o\left(r^{-1 / 2}\right) \quad \text { as } \quad r \rightarrow \infty
$$

uniformly for all directions, where $\Omega$ is an unbounded domain in the plane $R^{2}$ with boundary $\Gamma$ which is a closed smooth curve, $\operatorname{Im}(k)>0, i=\sqrt{-1}, r=\sqrt{x^{2}+y^{2}}$ and $n$ is the outer normal to $\Gamma$.

The coupled procedure in [1] and [2] are based upon the direct BEM in which the boundary integral equations come from Green's formula, and the unknowns on the common boundary involve $\frac{\partial u}{\partial n}$ as well as $u$. Feng and Yu (cf. [3], [4]) developed an integral equation for the Laplace equation with a circular boundary by using Green's function. Here we also take a circle as the common boundary,

Received by the editor November 21, 1996 and, in revised form, April 10, 1997 and January $22,1998$.

1991 Mathematics Subject Classification. Primary 65N38, 65N30, 15A06.

Key words and phrases. BEM, FEM, Helmholtz equation, integral equation, Fourier expansion, variational equation.

(C)1999 American Mathematical Society 
but an integral equation which is called the Dirichlet to Neumann (DtN) boundary condition in [5] is obtained by Fourier expansion. As a result, only $u$ is considered unknown on the circle. Such a procedure is advantageous from a numerical point of view. In Section 4, we give a convergence analysis which is different from one for $k=0$ in [5] and $k>0$ in [6].

\section{Integral EQUATion on a Circle}

In order to obtain an integral equation on a circle, assume the boundary $\Gamma$ in this section is a circle of radius $R$ whose centre is the origin of coordinates. In the polar coordinates system $r, \theta$ in the plane, the equation (1.1a) and the boundary conditions (1.1b) and (1.2b) become, respectively,

$$
\begin{gathered}
\frac{\partial^{2} u}{\partial r^{2}}+\frac{1}{r} \frac{\partial u}{\partial r}+\frac{1}{r^{2}} \frac{\partial^{2} u}{\partial \theta^{2}}+k^{2} u=0 \\
u(R, \theta)=u_{0}(\theta)
\end{gathered}
$$

and

$$
\frac{\partial u}{\partial n}(R, \theta)=u_{n}(\theta)
$$

From the periodicity of the boundary conditions, the solution of equation (2.1) is a $2 \pi$-periodic function for $\theta$ and can be expressed as a Fourier series. We find that the function

$$
u(r, \theta)=\sum_{n=-\infty}^{\infty} c_{n} H_{|n|}^{(1)}(k r) e^{i n \theta}, \quad r>R,
$$

satisfies equation (2.1) and the radiation condition (1.3), where $H_{n}^{(1)}(z)$ denotes the Hankel function of the first kind

$$
H_{n}^{(1)}(z)=J_{n}(z)+i Y_{n}(z), \quad n=0,1,2, \ldots,
$$

and $J_{n}(z)$ and $Y_{n}(z)$ being the Bessel functions of the first and second kind, respectively:

$$
\begin{aligned}
J_{n}(z)= & \sum_{j=0}^{\infty}(-1)^{j} \frac{1}{j !(j+n) !}\left(\frac{z}{2}\right)^{2 j+n}, \quad n=0,1,2, \ldots, \\
Y_{n}(z)= & \frac{2}{\pi} J_{n}(z) \ln \frac{z}{2}-\frac{1}{\pi} \sum_{j=0}^{n-1} \frac{(n-j-1) !}{j !}\left(\frac{z}{2}\right)^{2 j-n} \\
& -\frac{1}{\pi} \sum_{j=0}^{\infty}(-1)^{j} \frac{1}{j !(j+n) !}[\psi(n+j+1) \\
& +\psi(j+1)]\left(\frac{z}{2}\right)^{2 j+n}, \quad n=0,1,2, \ldots,
\end{aligned}
$$

with

$$
|\arg (z)|<\pi, \quad \psi(1)=-\gamma, \quad \psi(m)=-\gamma+1+\frac{1}{2}+\cdots+\frac{1}{m-1}, \quad m \geq 2,
$$

in which $\gamma=0.5772156649 \ldots$ is Euler's constant. The coefficient $c_{n}$ in (2.4) will be determined from the boundary conditions.

Substituting the boundary condition (2.2) into (2.4), we have

$$
u(r, \theta)=\sum_{n=-\infty}^{\infty} a_{n} G_{|n|}(k R, k r) e^{i n \theta}, \quad r>R,
$$


where $a_{n}$ is the Fourier coefficient for $u_{0}(\theta)$

$$
a_{n}=\frac{1}{2 \pi} \int_{0}^{2 \pi} u_{0}(\theta) e^{-i n \theta} d \theta, \quad G_{n}(x, y)=H_{n}^{(1)}(y) / H_{n}^{(1)}(x) .
$$

From (2.6) and (2.7) we find that the asymptotic behavior of $H_{n}^{(1)}(z)$ is given by

$$
H_{n}^{(1)}(z)=-i \frac{(n-1) !}{\pi}\left(\frac{z}{2}\right)^{-n}\left(1+\frac{1}{n-1}\left(\frac{z}{2}\right)^{2}+O\left(\frac{1}{n^{2}}\right)\right) \quad \text { as } n \rightarrow \infty
$$

and furthermore

$$
G_{n}(k R, k r)=\left(\frac{R}{r}\right)^{n}\left(1+O\left(\frac{1}{n}\right)\right) \quad \text { as } n \rightarrow \infty .
$$

Hence the series in (2.8) is absolutely and uniformly convergent on any closed interval in $(R, \infty)$ and can be differentiated term by term, and the function $u(r, \theta)$ in (2.8) is indeed the solution for the Dirichlet problem (1.1a), (2.2) with the radiation condition (1.3). Introducing the integral operator $P$ defined by

$$
P v(\theta)=\frac{1}{2 \pi} \int_{0}^{2 \pi}\left[G_{0}(k R, k r)+2 \sum_{n=1}^{\infty} G_{n}(k R, k r) \cos n\left(\theta-\theta^{\prime}\right)\right] v\left(\theta^{\prime}\right) d \theta^{\prime}, \quad r>R,
$$

the formula (2.8) can be rewritten as

$$
u(r, \theta)=P u_{0}(\theta), \quad r>R .
$$

Differentiating (2.12) for $r$ and letting $r \rightarrow R+0$, we get a boundary integral equation

$$
K u(R, \theta)=\frac{\partial u}{\partial n}(R, \theta)
$$

where $K$ is the boundary integral operator defined by

$$
K v(\theta)=\frac{k}{2 \pi} \int_{0}^{2 \pi}\left[H_{0}(k R)+2 \sum_{n=1}^{\infty} H_{n}(k R) \cos n\left(\theta-\theta^{\prime}\right)\right] v\left(\theta^{\prime}\right) d \theta^{\prime}
$$

with

$$
H_{n}(z)=-\frac{d H_{n}^{(1)}(z)}{d z} / H_{n}^{(1)}(z)=\frac{H_{n+1}^{(1)}(z)}{H_{n}^{(1)}(z)}-\frac{n}{z}, \quad n=0,1,2, \ldots
$$

From (2.9), we find that

$$
H_{n}(z)=\frac{n}{z}\left(1-\frac{2}{n(n-1)}\left(\frac{z}{2}\right)^{2}+O\left(\frac{1}{n^{4}}\right)\right) \quad \text { as } n \rightarrow \infty
$$

and the series in (2.14) is divergent. Since

$$
\sum_{n=1}^{\infty} \frac{1}{n} \cos n \theta=-\ln \left|2 \sin \frac{\theta}{2}\right|, \quad \theta \neq 2 m \pi,
$$

and since in the theory of generalized functions $([7])$

$$
\sum_{n=1}^{\infty} n \cos n \theta=-\frac{1}{4 \sin ^{2} \frac{\theta}{2}}, \quad \theta \neq 2 m \pi,
$$

it follows that the integral kernel in (2.14) contains a singularity of the $\left(\theta-\theta^{\prime}\right)^{-2}$ type and the integral should be considered as the finite part of the Hadamard hypersingular integral ([3], [4], [8]). 
Similarly, we can obtain the solution for the Neumann problem (1.2a),(2.3),(1.3)

$$
\begin{array}{r}
u(r, \theta)=\frac{1}{2 \pi k} \int_{0}^{2 \pi}\left[\frac{G_{0}(k R, k r)}{H_{0}(k R)}+2 \sum_{m=1}^{\infty} \frac{G_{m}(k R, k r)}{H_{m}(k R)} \cos m\left(\theta-\theta^{\prime}\right)\right] u_{n}\left(\theta^{\prime}\right) d \theta^{\prime} \\
r \geq R,
\end{array}
$$

and another boundary integral equation

$$
\frac{1}{2 \pi k} \int_{0}^{2 \pi}\left[\frac{1}{H_{0}(k R)}+2 \sum_{m=1}^{\infty} \frac{1}{H_{m}(k R)} \cos m\left(\theta-\theta^{\prime}\right)\right] \frac{\partial u}{\partial n}\left(R, \theta^{\prime}\right) d \theta^{\prime}=u(R, \theta)
$$

whose kernel contains an integrable singularity of the $\ln \left|\theta-\theta^{\prime}\right|$ type.

\section{Coupled procedure AND VARIATIONAL FORMULATion}

Now we discuss the coupled procedure of BEM and FEM. Make a circle $\Gamma_{0}=$ $\left\{(x, y) \mid x^{2}+y^{2}=R^{2}\right\}$ for an appropriate radius $R$ such that the boundary $\Gamma$ is surrounded by $\Gamma_{0}$, and let $\Gamma_{0}$ divide the domain $\Omega$ into a bounded part $\Omega_{1}=$ $\left\{(x, y) \mid x^{2}+y^{2}<R^{2},(x, y) \in \Omega\right\}$ and an unbounded part $\Omega_{2}=\left\{(x, y) \mid x^{2}+y^{2}>R^{2}\right\}$. From the last section we see that the function $u$ satisfying equation (1.1a) in $\Omega_{2}$ and the radiation condition (1.3) satisfies

$$
\frac{\partial u}{\partial n}=K\left(\gamma_{0} u\right) \quad \text { on } \Gamma_{0}
$$

with the trace operator $\gamma_{0}$ defined by $\gamma_{0} u=\left.u\right|_{\Gamma_{0}}$. Thus the exterior Dirichlet problem (1.1),(1.3) and the exterior Neumann problem (1.2),(1.3) are transformed into

$$
\begin{cases}\triangle u+k^{2} u=0 & \text { in } \Omega_{1} \\ u=u_{0} & \text { on } \Gamma, \\ \frac{\partial u}{\partial n}=K\left(\gamma_{0} u\right) & \text { on } \Gamma_{0}\end{cases}
$$

and

$$
\begin{cases}\triangle u+k^{2} u=0 & \text { in } \Omega_{1} \\ \frac{\partial u}{\partial n}=u_{n} & \text { on } \Gamma \\ \frac{\partial u}{\partial n}=K\left(\gamma_{0} u\right) & \text { on } \Gamma_{0}\end{cases}
$$

respectively.

Now we can apply FEM to the nonlocal boundary value problems (3.1) and (3.2) in the bounded domain $\Omega_{1}$. The variational problem corresponding to (3.2) is: Find $u \in H^{1}\left(\Omega_{1}\right)$ such that

$$
A(u, v)=\left\langle u_{n}, v\right\rangle \quad \forall v \in H^{1}\left(\Omega_{1}\right)
$$

where

$$
\begin{aligned}
& A(u, v)=a_{1}(u, v)+b\left(\gamma_{0} u, \gamma_{0} v\right), \\
& a_{1}(u, v)=\int_{\Omega_{1}}\left(\nabla u \cdot \nabla v-k^{2} u v\right) d \Omega, \quad b\left(\gamma_{0} u, \gamma_{0} v\right)=\int_{\Gamma_{0}}\left(\gamma_{0} v\right) K\left(\gamma_{0} u\right) d s, \\
& \left\langle u_{n}, v\right\rangle=\int_{\Gamma} u_{n} v d s, \quad \nabla u \cdot \nabla v=\frac{\partial u}{\partial x} \frac{\partial v}{\partial x}+\frac{\partial u}{\partial y} \frac{\partial v}{\partial y} .
\end{aligned}
$$

Obviously, $a_{1}(\cdot, \cdot), b(\cdot, \cdot)$ and $A(\cdot, \cdot)$ are symmetric bilinear forms. 
It must be pointed out that the unknown $\gamma_{0}\left(\frac{\partial u}{\partial n}\right)$ is not involved in (3.3). This is why we use the integral equation (2.13) on $\Gamma_{0}$, not (2.17), and select a circle as the common boundary, not another closed curve. This coupled procedure is superior to that based upon the direct BEM.

For the following discussion, we introduce the function spaces

$$
\begin{aligned}
& H_{l o c}^{1}(\Omega)=\left\{u \mid u \in H^{1}(\tilde{\Omega}) \quad \text { for any compact set } \tilde{\Omega} \subset \Omega\right\}, \\
& H_{r}^{1}(\Omega)=\left\{u \mid u \in H_{l o c}^{1}(\Omega), u \text { satisfying the radiation condition }(1.3)\right\}, \\
& H_{L}^{1}(\Omega)=\left\{u \mid u \in H_{r}^{1}(\Omega), \triangle u+k^{2} u=0 \text { in } \Omega\right\}, \\
& H_{c}^{1}(\Omega)=\left\{u \mid u \in H_{l o c}^{1}(\Omega), u \text { having compact support in } \Omega\right\},
\end{aligned}
$$

and define

$$
a_{2}(u, v)=\int_{\Omega_{2}}\left(\nabla u \cdot \nabla v-k^{2} u v\right) d \Omega, \quad a(u, v)=a_{1}(u, v)+a_{2}(u, v) .
$$

Lemma 3.1.

$$
b\left(\gamma_{0} u, \gamma_{0} v\right)=a_{2}(u, v) \quad \forall u \in H_{L}^{1}\left(\Omega_{2}\right), v \in H_{c}^{1}\left(\Omega_{2}\right) .
$$

Proof. It follows from Green's formula.

Theorem 3.1. Let $u_{n} \in H^{-1 / 2}(\Gamma)$ be given. Thus the variational problem (3.3) in $\Omega_{1}$ is equivalent to the variational problem corresponding to (1.2), (1.3) in $\Omega$ : Find $u \in H_{r}^{1}(\Omega)$ such that

$$
a(u, v)=\left\langle u_{n}, v\right\rangle \quad \forall v \in H_{c}^{1}(\Omega) .
$$

In other words, if $u_{1} \in H^{1}\left(\Omega_{1}\right)$ is the solution of (3.3), then

$$
u= \begin{cases}u_{1} & \text { in } \bar{\Omega}_{1} \\ u_{2} \equiv P\left(\gamma_{0} u_{1}\right) & \text { in } \Omega_{2}\end{cases}
$$

is the solution of (3.6); conversely, if $u \in H_{r}^{1}(\Omega)$ is the solution of (3.6), then $u$ in $\Omega_{1}$ is the solution of (3.3).

Proof. Let $u_{1} \in H^{1}\left(\Omega_{1}\right)$ satisfy the variational formula (3.3). So $u_{2} \equiv P\left(\gamma_{0} u_{1}\right) \in$ $H_{L}^{1}\left(\Omega_{2}\right), \gamma_{0} u_{2}=\gamma_{0} u_{1} \in H^{1 / 2}\left(\Gamma_{0}\right)$ and $u \in H_{r}^{1}(\Omega)$. From Lemma 3.1, it follows that

$$
a(u, v)=a_{1}(u, v)+b\left(\gamma_{0} u, \gamma_{0} v\right)=A\left(u_{1}, v\right)=\left\langle u_{n}, v\right\rangle \quad \forall v \in H_{c}^{1}(\Omega) .
$$

Hence $u$ is the solution of (3.6).

Conversely, assume $u \in H_{r}^{1}(\Omega)$ satisfies the variational formula (3.6); then $u$ is the generalized solution of $(1.2),(1.3)$. For any $v_{1} \in H^{1}\left(\Omega_{1}\right)$, it is known that $\gamma_{0} v_{1} \in H^{1 / 2}\left(\Gamma_{0}\right)$, and there exists $v_{2} \in H_{c}^{1}\left(\Omega_{2}\right)$ such that $\gamma_{0} v_{2}=\gamma_{0} v_{1}$ from the trace theorem. Writing

$$
v= \begin{cases}v_{1} & \text { in } \bar{\Omega}_{1} \\ v_{2} & \text { in } \Omega_{2},\end{cases}
$$

we see that $v \in H_{c}^{1}(\Omega)$, and

$$
A\left(u, v_{1}\right)=a_{1}\left(u, v_{1}\right)+a_{2}\left(u, v_{2}\right)=a(u, v)=\left\langle u_{n}, v\right\rangle=\left\langle u_{n}, v_{1}\right\rangle .
$$

This shows that $u$ in $\Omega_{1}$ is the solution of (3.3). 
From the solvability and uniqueness of (3.6), now we can say that for any given $u_{n} \in H^{-1 / 2}(\Gamma)$ there exists exactly one variational solution to (3.3).

In the following discussion, $\|\cdot\|_{s, D}$ denotes the norm in the Sobolev space $H^{s}(D)$ and $\|\cdot\|_{0, D}$ is $L^{2}(D)$-norm for $s=0$.

Lemma 3.2. The linear operator $K: H^{s}\left(\Gamma_{0}\right) \rightarrow H^{s-1}\left(\Gamma_{0}\right)$ is continuous, i.e.

$$
\|K f\|_{s-1, \Gamma_{0}} \leq c\|f\|_{s, \Gamma_{0}} \quad \forall f \in H^{s}\left(\Gamma_{0}\right)
$$

where $s$ is any real number and $c$ is a positive constant independent of $f$.

Proof. Let $f(\theta) \in H^{s}\left(\Gamma_{0}\right)$ and expand it in the Fourier series

$$
f(\theta)=\sum_{n=-\infty}^{\infty} a_{n} e^{i n \theta}
$$

with the Fourier coefficients

$$
a_{n}=\frac{1}{2 \pi} \int_{0}^{2 \pi} f(\theta) e^{-i n \theta} d \theta
$$

The norm in $H^{s}\left(\Gamma_{0}\right)$ can be defined using the Fourier coefficiets as

$$
\|f\|_{s, \Gamma_{0}}=\left[2 \pi R \sum_{n=-\infty}^{\infty}\left(n^{2}+1\right)^{s}\left|a_{n}\right|^{2}\right]^{1 / 2} .
$$

Thus from (2.14) we have

$$
\begin{gathered}
K f=k \sum_{-\infty}^{\infty} a_{n} H_{|n|}(k R) e^{i n \theta}, \\
\|K f\|_{s-1, \Gamma_{0}}^{2}=2 \pi|k|^{2} R \sum_{-\infty}^{\infty}\left(n^{2}+1\right)^{s-1}\left|a_{n} H_{|n|}(k R)\right|^{2} .
\end{gathered}
$$

By (2.16), there exists a positive integer $N$ such that $\frac{1}{2}<\left|\frac{k R}{n} H_{n}(k R)\right|<\frac{3}{2}$ when $n>N$, and we obtain (3.7) with

$$
c=\max \left\{\frac{3}{2 R}, \max _{0 \leq n \leq N}\left\{\left|k H_{n}(k R)\right| / \sqrt{n^{2}+1}\right\}\right\} .
$$

Theorem 3.2. The symmetric bilinear form $b(\cdot, \cdot)$ on the space $H^{1 / 2}\left(\Gamma_{0}\right) \times$ $H^{1 / 2}\left(\Gamma_{0}\right)$ has the following properties:

1. $|b(f, g)| \leq c_{1}\|f\|_{1 / 2, \Gamma_{0}}\|g\|_{1 / 2, \Gamma_{0}}$ (continuity).

2. $\operatorname{Im}(-\bar{k} b(f, \bar{f})) \geq c_{2}\|f\|_{1 / 2, \Gamma_{0}}^{2}\left(H^{1 / 2}\left(\Gamma_{0}\right)\right.$-coercivity $)$.

Here $f, g \in H^{1 / 2}\left(\Gamma_{0}\right)$ are arbitrary functions and $c_{1}, c_{2}>0$ are constants independent of $f$ and $g$.

Proof. (1) It comes from Lemma 3.2 that

$$
|b(f, g)|=\left|\int_{\Gamma_{0}} g K f d s\right| \leq\|g\|_{1 / 2, \Gamma_{0}}\|K f\|_{-1 / 2, \Gamma_{0}} \leq c_{1}\|f\|_{1 / 2, \Gamma_{0}}\|g\|_{1 / 2, \Gamma_{0}} .
$$

(2) Let

$$
u(r, \theta)=\operatorname{Pf}(\theta)
$$


Thus $u \in H_{L}^{1}\left(\Omega_{2}\right), \gamma_{0} u=f$. Defining $\Omega_{d}=\left\{(x, y) \mid R^{2}<x^{2}+y^{2}<d^{2}\right\}$ and $\Gamma_{d}=\left\{(x, y) \mid x^{2}+y^{2}=d^{2}\right\}$ for $d>R$ and using the radiation condition and Green's formula, we have

$$
\begin{aligned}
& 0=\lim _{d \rightarrow \infty} \int_{\Gamma_{d}}\left|\frac{\partial u}{\partial r}-i k u\right|^{2} d s=\lim _{d \rightarrow \infty} \int_{\Gamma_{d}}\left[\left|\frac{\partial u}{\partial r}\right|^{2}+|k u|^{2}-2 \operatorname{Im}\left(\bar{k} \bar{u} \frac{\partial u}{\partial r}\right)\right] d s, \\
& \int_{\Gamma_{d}} \bar{u} \frac{\partial u}{\partial r} d s=-\int_{\Omega_{d}}\left(k^{2}|u|^{2}-|\nabla u|^{2}\right) d \Omega-\int_{\Gamma_{0}} \bar{u} \frac{\partial u}{\partial n} d s .
\end{aligned}
$$

Combine them to give

$$
\begin{aligned}
\operatorname{Im}(-\bar{k} b(f, \bar{f})) & =\operatorname{Im}\left(-\bar{k} \int_{\Gamma_{0}} \bar{u} \frac{\partial u}{\partial n} d s\right) \\
& =\lim _{d \rightarrow \infty}\left[\frac{1}{2} \int_{\Gamma_{d}}\left(\left|\frac{\partial u}{\partial r}\right|^{2}+|k u|^{2}\right) d s+\operatorname{Im}(k) \int_{\Omega_{d}}\left(|\nabla u|^{2}+|k u|^{2}\right) d \Omega\right]
\end{aligned}
$$

If $\operatorname{Im}(k)>0$, from the trace theorem we get

$$
\operatorname{Im}(-\bar{k} b(f, \bar{f})) \geq \operatorname{Im}(k) \min \left\{1,|k|^{2}\right\}\|u\|_{1, \Omega_{2}}^{2} \geq c_{2}\|f\|_{1 / 2, \Gamma_{0}}^{2} .
$$

Theorem 3.2 can also be proved like Lemma 3.2, and from Theorem 3.2 we obtain the following theorem.

Theorem 3.3. The symmetric bilinear form $A(\cdot, \cdot)$ on the space $H^{1}\left(\Omega_{1}\right) \times H^{1}\left(\Omega_{1}\right)$ has the following properties:

1. $|A(u, v)| \leq c_{1}\|u\|_{1, \Omega_{1}}\|v\|_{1, \Omega_{1}}$ (continuity).

2. $\operatorname{Im}(-\bar{k} A(u, \bar{u})) \geq c_{2}\|u\|_{1, \Omega_{1}}^{2}\left(H^{1}\left(\Omega_{1}\right)\right.$-coercivity $)$.

Here $u, v \in H^{1}\left(\Omega_{1}\right)$ are arbitrary functions and $c_{1}, c_{2}>0$ are constants independent of $u$ and $v$.

Theorem 3.3 also shows that the variational problem (3.3) has only one solution. By (3.8) and Theorem 3.2, there exists a constant $c>0$ such that

$$
\|P f\|_{1, \Omega_{2}} \leq c\|f\|_{1 / 2, \Gamma_{0}} \quad \forall f \in H^{1 / 2}\left(\Gamma_{0}\right) .
$$

The above discussion can be applied to the boundary value problem (3.1), in which the boundary condition on $\Gamma$ is essential.

\section{Convergence analysis And error estimate}

In the previous section a boundary value problem in an unbounded domain has been transformed into the corresponding variational problem in a bounded one. Now FEM can be applied in $\Omega_{1}$. Let $\psi_{1}(x, y), \ldots, \psi_{M}(x, y)$ be the local base functions of interpolation in FEM, and $u^{h}(x, y)$ be the interpolation function for $u(x, y)$ on $\bar{\Omega}_{1}$ defined by

$$
u^{h}(x, y)=\sum_{j=1}^{M} u_{j} \psi_{j}(x, y) \quad \text { in } \quad \bar{\Omega}_{1} .
$$

So the discrete variational problem corresponding to (3.3) is: Find $u^{h} \in S^{h}\left(\Omega_{1}\right)$ such that

$$
A\left(u^{h}, v^{h}\right)=\left\langle u_{n}, v^{h}\right\rangle \quad \forall v^{h} \in S^{h}\left(\Omega_{1}\right)
$$


where $S^{h}\left(\Omega_{1}\right)$ is the finite element space defined by

$$
S^{h}\left(\Omega_{1}\right)=\operatorname{Span}\left\{\psi_{1}, \ldots, \psi_{M}\right\} \subset H^{1}\left(\Omega_{1}\right) .
$$

In the following, $u$ and $u^{h}$ denote the solutions for the variational problem (3.3) and the discrete variational problem (4.2), respectively, $h$ is the maximal length of the diameters of elements, $\Pi: H^{1}\left(\Omega_{1}\right) \rightarrow S^{h}\left(\Omega_{1}\right)$ is the interpolation operator, and $c$ denotes a positive constant independent of $u$ and $u^{h}$; it can have different values at different places.

Theorem 4.1. If $u \in H^{j+1}\left(\Omega_{1}\right)$ and

$$
\|v-\Pi v\|_{1, \Omega_{1}} \leq c h^{j}\|v\|_{j+1, \Omega_{1}} \quad \forall v \in H^{j+1}\left(\Omega_{1}\right),
$$

then

$$
\left\|u-u^{h}\right\|_{1, \Omega_{1}} \leq c h^{j}\|u\|_{j+1, \Omega_{1}} .
$$

Proof. By Theorem 3.3, we use Céa's lemma to obtain

$$
\left\|u-u^{h}\right\|_{1, \Omega_{1}} \leq c\|u-\Pi u\|_{1, \Omega_{1}} \leq c h^{j}\|u\|_{j+1, \Omega_{1}} .
$$

\section{Theorem 4.2.}

$$
\begin{aligned}
& \left|P\left(\gamma_{0} u\right)-P\left(\gamma_{0} u^{h}\right)\right| \leq c_{r}\left\|u-u^{h}\right\|_{0, \Gamma_{0}} \leq c_{r}\left\|u-u^{h}\right\|_{1, \Omega_{1}}, \quad r>R, \\
& \left\|P\left(\gamma_{0} u\right)-P\left(\gamma_{0} u^{h}\right)\right\|_{1, \Omega_{2}} \leq c\left\|u-u^{h}\right\|_{1 / 2, \Gamma_{0}} \leq c\left\|u-u^{h}\right\|_{1, \Omega_{1},}
\end{aligned}
$$

where $c_{r}$ depends on $r$.

Proof. By (2.11),

$$
\begin{aligned}
\left|P\left(\gamma_{0} u\right)-P\left(\gamma_{0} u^{h}\right)\right| & =\frac{1}{2 \pi}\left|\int_{0}^{2 \pi}\left[G_{0}+2 \sum_{n=1}^{\infty} G_{n} \cos n\left(\theta-\theta^{\prime}\right)\right]\left(\gamma_{0} u-\gamma_{0} u^{h}\right) d \theta^{\prime}\right| \\
& \leq \frac{1}{2 \pi}\left[2 \pi\left(\left|G_{0}\right|^{2}+2 \sum_{n=1}^{\infty}\left|G_{n}\right|^{2}\right) \int_{0}^{2 \pi}\left|\gamma_{0} u-\gamma_{0} u^{h}\right|^{2} d \theta^{\prime}\right]^{1 / 2} \\
& =\left[\frac{1}{2 \pi R}\left(\left|G_{0}\right|^{2}+2 \sum_{n=1}^{\infty}\left|G_{n}\right|^{2}\right)\right]^{1 / 2}\left\|u-u^{h}\right\|_{0, \Gamma_{0}},
\end{aligned}
$$

and the series is convergent for $r>R$ by (2.10), and (4.3) is proved by the trace theorem. We use (3.9) and the trace theorem to arrive at (4.4).

The last theorem shows that the errors in $\Omega_{2}$ can be controlled by the errors on $\Gamma_{0}$ and in $\Omega_{1}$.

For time-harmonic acoustic wave propagation in a homogeneous isotropic medium, $k^{2}=\omega(\omega+i \gamma) / c^{2}$, where $c$ is the speed of sound, $\omega$ is the frequency of the acoustic wave and $\gamma$ is the damping coefficient. If $|k|$ is large, the Helmholtz equation has a rapidly oscillating solution and the quality of a finite element solution depends significantly on the wavenumber $k$ as well as the stepwidth $h$ of meshes. Ihlenburg and Babuška [9] applied the FEM with piecewise linear approximation to a one-dimensional Helmholtz equation, and their results show that the relative error of the FE-solution in the $H^{1}$-seminorm is controlled by a term of order $k h$. Bayliss, et al., in [10] dealt with a two-dimentional Helmholtz equation with a real wavenumber $k>0$ in a bounded domain, and stated a convergence theorem which shows that the relative error bound is $O\left(k(k h)^{j+1}\right)$ in the $L^{2}$-norm and $O\left(k(k h)^{j}\right)$ in the $H^{1}$-norm, where $j$ is the order of polynomial approximation in FEM. In 
this paper, $\operatorname{Im}(k)>0$ in an unbounded domain is assumed. For a large $|k|$, the constants $c_{1}$ and $c_{2}$ in Theorem 3.3 are $|k|^{2}$ and $\operatorname{Im}(k)$, respectively, so the constant in Céa's lemma is of order $|k|^{3} / \operatorname{Im}(k)$. For a linear interpolation, the error of the FE-solution is

$$
\left\|u-u^{h}\right\|_{1, \Omega_{1}} \leq c \frac{|k|^{3}}{\operatorname{Im}(k)} h\|u\|_{2, \Omega_{1}},
$$

with a constant $c$ independent of $k$ and $h$. In addition, the constant $c$ in (4.4) is $\sqrt{|k| / \operatorname{Im}(k)}$.

\section{ACKNOWLEDGMENTS}

The author would like to thank the referees for their useful suggestions.

\section{REFERENCES}

1. C. Johnson and J. C. Nedelec, On the coupling of boundary integral and finite element methods, Math. Comp., 35(1980), pp.1063- 1079. MR 82c:65072

2. G. C. Hsiao, The coupling of BEM and FEM-a brief review, in Boundary Elements X, C. A. Brebbia et al., ed., Springer-Verlag, 1988, pp.431-445. MR 91c:65071

3. Feng Kang and Yu De-hao, Canonical integral equations of elliptic boundary value problems and their numerical solutions, in Proceedings of China-France Symposium on the Finite Element Method (1982, Beijing), Science Press, Beijing, 1983, pp.211-252.

4. Yu De-hao, A direct and natural coupling of BEM and FEM, in Boundary Elements XIII, C.A.Brebbia and G.S.Gipson ed., Computational Mechanics Publications, Southampton, 1991, pp.995-1004. MR 92h:65004

5. J. B. Keller and D. Givoli, Exact non-reflecting boundary conditions, J.Comp. Phys., 82(1989), pp.172-192. MR 91a:76064

6. A. K. Aziz and R. B. Kellogg, Finite element analysis of a scattering problem, Math. Comp., 37(1981), pp.261-272. MR 82i:65069

7. I. M. Gelfand and G. E. Shilov, Generalized Functions, Vol. 1, Academic Press, New York, 1964. MR 29:3869

8. V. J. Ervin, R. Kieser and W. L. Wendland, Numerical approximation of the solution for a model 2-D hypersingular integral equation, Universität Stuttgart, Math. Institut A, Bericht Nr.26, 1990; also in Computational Engineering with Boundary Elements, Vol. 1 (Proc. Fifth Internat. Conf., Newark, DE, 1990; S. Grilli et al., eds.), Comput. Mech. Publ., Southampton, 1990, pp. 85-99. MR 92i:65005

9. F. Ihlenburg and I. Babuška, Finite element solution of the Helmholtz equation with high wave number - part I: The h-version of the FEM, Comp. Math. Appl., 30(1995), No.9, pp.9-37. MR 96j:65123

10. A. Bayliss, C. I. Goldstein and E. Turkel, On accuracy conditions for the numerical computation of waves, J.Comp. Phys., 59(1985), pp.396-404. MR 87b:65153

Department of Mathematics, East China University of Science and Technology, Shanghai 200237, P.R.China 